\title{
Reliability design of mechanical systems subjected to repetitive stresses
}

\author{
Seong-woo $\mathrm{Woo}^{1 *}$, Dennis L. O’Neal ${ }^{2}$, and Yimer Mohammed Hassen ${ }^{1}$ \\ ${ }^{1}$ Dean of Mechanical Technology Faculty, Manufacturing Technology, Ethiopian Technical \\ University, Addis Ababa PO box 190310, Ethiopia \\ ${ }^{2}$ Department of Mechanical Engineering, Dean of Engineering and Computer Science, Baylor \\ University, Waco, TX 76798-7356, USA; dennis_oneal@baylor.edu
}

\begin{abstract}
To enhance the design of mechanical systems, parametric Accelerated Life Testing (ALT) as a systematic reliability method is proposed as a way to evaluate the design of mechanical systems subjected to repeated impact stresses. It requires: (1) a parametric ALT scheme shaped on system BX lifetime, (2) a load inspection, (3) parametric ALTs with the associated design modifications, and (4) an assessment of whether the revised product design(s) reach the targeted BX life-time. We propose using a general life-stress model and sample size equation. A test example using both market data and parametric ALT was the redesign of a hinge kit system (HKS) in a refrigerator. To conduct parametric ALTs, a force and moment balance analysis was utilized. The mechanical impact loadings of the HKS were evaluated for an working refrigerator door. For the first ALT, the HKS failure happened in the crack/fracture of the kit housing and oil spilled from the damper when the HKS was disassembled. The failure modes and mechanisms constructed in the 1st ALT were similar to those of the unsuccessful samples found from the marketplace. The missing design parameters of the HKS included stress raisers such as corner roundings and the rib of the housing in HKS, the seal in the oil damper, and the material of the cover housing. In the second ALT, the cover housing fractured. The design defect of the cover housing in the HKS was the plastic material. As a corrective action plan, the cover housing was modified from plastic to aluminium. After the second ALT, the lifetime of the modified HKS was reassured to be B1 life 10 years with a yearly failure rate of $0.1 \%$.
\end{abstract}

\section{Introduction}

Mechanical products such as automobiles, airplanes, and refrigerators convert some form of power into a mechanical advantages utilizing various mechanisms. In the process they are subjected to repetitive stresses. Most mechanical products are multi-module structures. If the modules are assembled, the mechanical product can satisfactorily function and perform its own planned purposes. For instance, a refrigerator is designed to provide chilled air from the evaporator to the freezer (or refrigerator). It includes various modules - cabinet, door, internal

* Corresponding author: twinwoo@yahoo.com 
fixture (shelves and drawers), generating parts (motor or compressor), controls and instruments, heat exchanger, water supply device, and other various parts. A refrigerator may have as many as 2,000 parts (Figure 1).

The reliability of a mechanical product might be described as the multiplication of its lifetime, $L_{B}$, and failure rate, $\lambda$. The entire failure rate of mechanical product such as refrigerator over its lifetime is the grand total of the failure rate of each module (module \#1: cabinet, module \#2: internal fixture). If there were no premature failures in a product, the product lifetime could be decided by problematic designed module \#3 in Figure 2 such as a newly designed HKS examined in case-study in this paper. The refrigerator lifetime is anticipated to beat a B20 life 10 years, That is, the time period that accumulated failure rate becomes $20 \%$ is ten years. If a refrigerator consists of 20 units and each unit has 100 components, the lifetime of each unit is targeted to be a B1 life 10 years. In other word, the time period that accumulated failure rate becomes $1 \%$ is ten years. We can conduct a parametric ALT for the newly designed mechanical system to potentially identify the design issues.

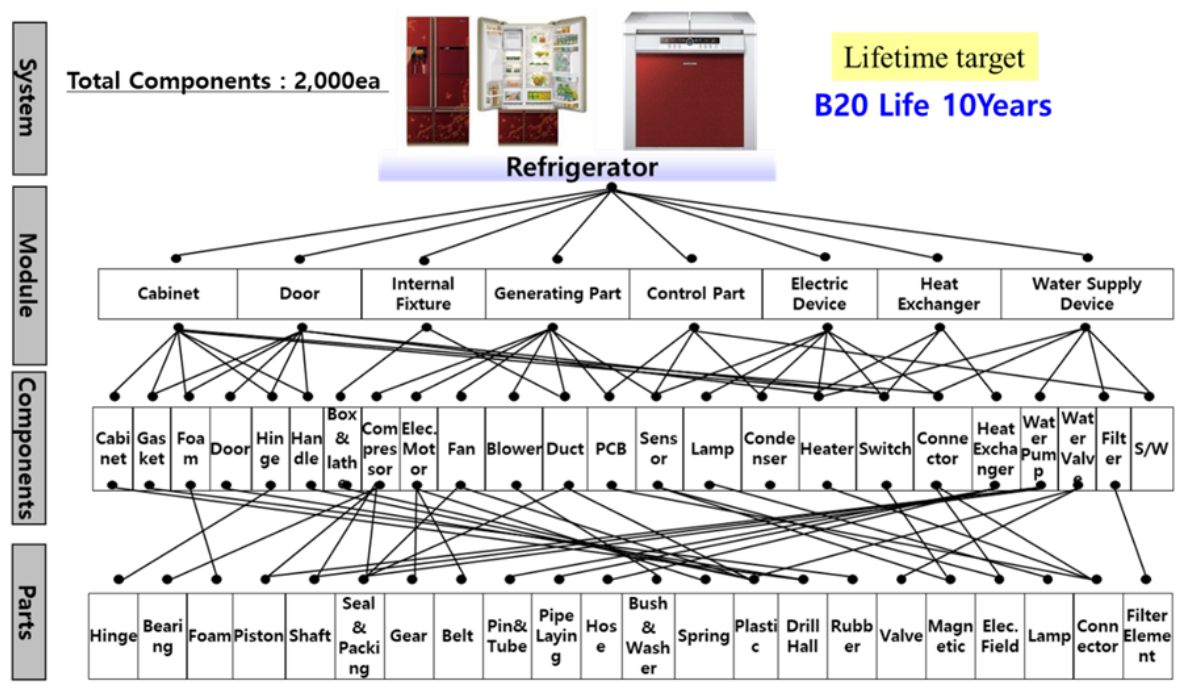

Fig. 1. Classification of refrigerator with multi-modules.

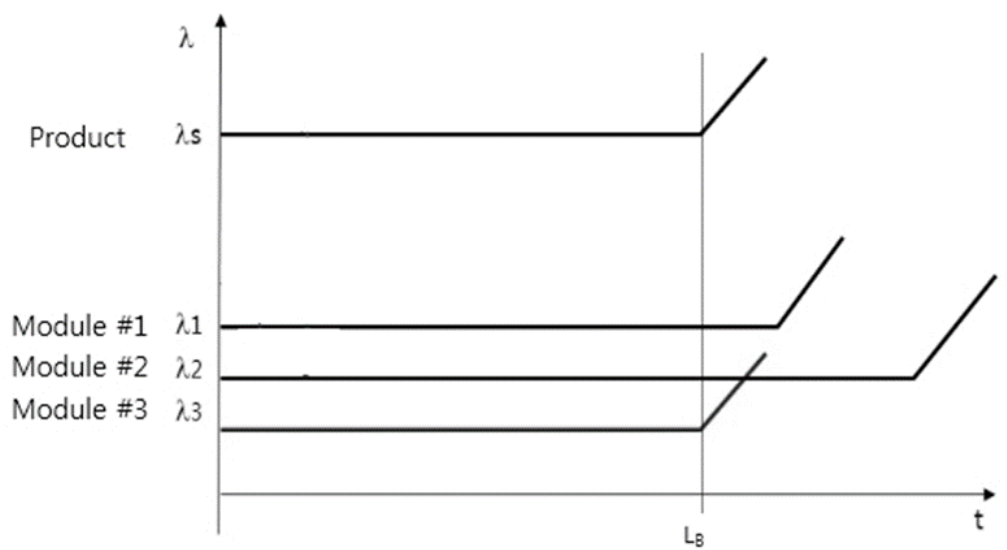

Fig. 2. Lifetime $L_{B}$ and failure rate $\lambda_{s}$ of multi-modules product. 
To reduce the failure rate of a mechanical system in the marketplace, it should be designed to robustly endure the working conditions for the consumers who purchase and use it. Any design defects should be identified and altered through statistical methodology or reliability testing [1] before a product is released. However, this approach demands enormous computations for an optimum answer but may not identify the most likely failure. If there are design faults that create an insufficiency of strength (or stiffness) when a product is subjected to repeated loads, the product will fail before its anticipated lifetime due to fatigue.

The typical methodologies for identifying failures in a product are stress-strength interference analysis, failure modes, effects, and criticality analysis (FMEA/FMECA), and fault tree analysis (FTA). In the product development process, these analyses are executed and documented by a company's technical specialists. Because the crucial designs of a new product is often missed in reviewing designs, the product can undergo field failures and then have to be recalled. Stress/strength interference models predict why mechanical products fail during a gradual wearout process. It also explains product failure if the stress is larger than the strength. However, because product failure may occur rapidly from fragile parts of a product, it necessitates using complementing design concepts such as fracture mechanics and life-stress model [2].

To execute the optimal design of a mechanical structure, engineers have investigated conventional design perspectives such as strength of materials. A new fracture mechanics study on the crucial components should be include fracture toughness instead of strength as a relevant material attribute. With quantum mechanics advances applied in electronic technology, engineers have identified system failures from micro-void coalescence (MVC) that may appear in metallic alloys or numerous engineered plastics. To determine the failure phenomena of a mechanical product, a better life-stress model might be combined with the traditional design approaches and applicable to electronic parts identifying a small crack or pre-existing defect that is impractical to model using FEM.

To understand why systems failure in the field, some engineers try to use the finite element method (FEM) to model the components in a system. Many engineers believe that infrequent product failures can be appraised by: (1) mathematical modeling utilizing Newtonian or Lagrangian methods, (2) after getting the system response for (dynamic) loads, the product stress/strain from it obtains, (3) employing the rain-flow counting method for von Mises stress, and (4) approximating the system damage by the Palmgren-Miner's law. However, employing a systematic method that can produce a closed-form, specific answers would entail making countless assumptions that cannot identify multi-module product failures due to micro-void, contacts, de-sign defects, etc. when subjected to loads.

This work presents a parametric ALT as a systematic reliability method that might be relevant to mechanical systems. It contains: (1) a parametric ALT scheme shaped on product BX lifetime, (2) a load inspection for ALT, (3) a parametric ALTs with the design changes, and (4) an assessment of whether the latest design(s) of the product gets the objective BX lifetime. As a case-study, we will examine the design of a HKS in a domestic refrigerator.

\section{Parametric ALT for Mechanical System}

\subsection{Placing an comprehensive testing plan}

Reliability can be manifested as the system potential to run under stated conditions for a specified period of time. Product reliability can be illustrated by a diagram called the "bathtub curve" that is composed of three areas. At the beginning, there is a lessening failure rate in 
the early life of the product $(\beta<1)$. Then, there is a nearly constant failure rate $(\beta=1)$. Ultimately, there is a growing failure rate at the end of the system's life $(\beta>1)$. If a product follows the bathtub curve, it may have difficulties being successful in the field because of the large failure rates and short lifetimes due to design defects. A manufacturer can update the product design by targeting reliability for new products to (1) minimizing premature failures, (2) reduce random failures during the product working period, and (3) enlarge product lifetime. As the design of a mechanical product improves, the failure rate of the product in the market decreases and its lifetime lengthens. For such states, the failure rate can become nearly a straight line (Figure 3 ).

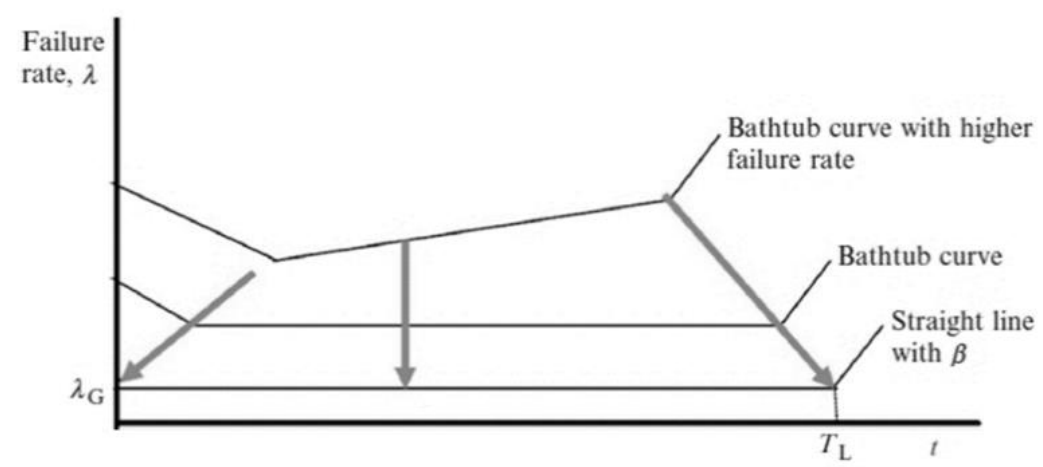

Fig. 3. Bathtub curve and straight line with slope $\beta$ to the ending of the life of the product

For a constant failure rate, the cumulative failure function of a mechanical product might be evaluated from the product lifetime LB and failure rate $\lambda$ as follows:

$$
F\left(L_{B}\right)=1-\mathrm{R}\left(\mathrm{L}_{\mathrm{B}}\right)=1-\mathrm{e}^{-\lambda \mathrm{L}_{\mathrm{B}}} \cong \lambda \mathrm{L}_{\mathrm{B}}
$$

where $\mathrm{R}(\cdot)$ is reliability function, $\mathrm{F}(\cdot)$ is unreliability function.

Equation (1) is relevant to $\leq 20 \%$ of cumulative failures, $\mathrm{F}(\cdot)$. After targeting the product lifetime $L_{B}$, designer should recognize any design defects and alter them through parametric ALT.

\subsection{Failure mechanism and accelerating testing}

Mechanical systems move (generated) power from one place to another by adapting its mechanisms. It will be subjected to the repeated loads over its lifetime and endure them so that it can reach the targeted reliability. The most important matter thus is how a potential premature failure mode can be identified through testing. A life-stress (LS) model of the mechanical system can be developed which involve stresses and reaction parameters. This model can describe some failures such as fatigue in the mechanical system. Fatigue failure appears, not due to conceptual stresses in a flawless part, but rather due to the existing of a tiny crack or a defect on the exterior of a part that can become plastic by the implied stress. It is important to understand how a small crack or pre-existing material defects may be generated.

Because system failures start from the existence of a material defect shaped on a microscopic level, we might evaluate the life-stress model from such a standpoint. For example, we could adopt processes utilized for solid-state diffusion of impurities in silicon that is widely used as semi-conduct material: 1) electro-migration-induced voiding; 2) build- 
up of chloride ions; and 3) trapping of electrons or holes. The generalized life-stress model might be stated as [1]:

$$
T F=A[\sinh (a S)]^{-1} \exp \left(\frac{E_{a}}{k T}\right)
$$

The hyperbolic sine stress term grows the stress as follows: (1) initially $(S)^{-1}$ in low stress effect, (2) $(S)^{-n}$ in medium stress effect, and (3) $\left(e^{a S}\right)^{-1}$ in high stress effect. Because ALT will be executed in the medium stress range, Equation (2) is stated as follows:

$$
T F=A(S)^{-n} \exp \left(\frac{E_{a}}{k T}\right)
$$

Because the stress of a mechanical system is hard to measure in testing, we need to redefine Equation (3). When the power is expressed as the multiplication of effort and flows, stresses may come from effort in a multi-port system. Equation (3) can then be stated as the more general form:

$$
T F=A(S)^{-n} \exp \left(\frac{E_{a}}{k T}\right)=A(e)^{-\lambda} \exp \left(\frac{E_{a}}{k T}\right)
$$

Design defects in products can be attained by exerting larger forces under elevated conditions. From the time-to-failure in Equation (4), an acceleration factor (AF) can be stated as the proportion between the proper elevated condition levels and common condition levels. AF can be stated to integrate the effort ideas:

$$
A F=\left(\frac{S_{1}}{S_{0}}\right)^{n}\left[\frac{E_{a}}{k}\left(\frac{1}{T_{0}}-\frac{1}{T_{1}}\right)\right]=\left(\frac{e_{1}}{e_{0}}\right)^{\lambda}\left[\frac{E_{a}}{k}\left(\frac{1}{T_{0}}-\frac{1}{T_{1}}\right)\right]
$$

Sample size equation with the AF in Equation (5) would be stated as [2]:

$$
n \geq(r+1) \cdot \frac{1}{x} \cdot\left(\frac{L_{B X}^{*}}{A F \cdot h_{a}}\right)^{\beta}+r
$$

\subsection{Case study: improving the lifetime of the HKS}

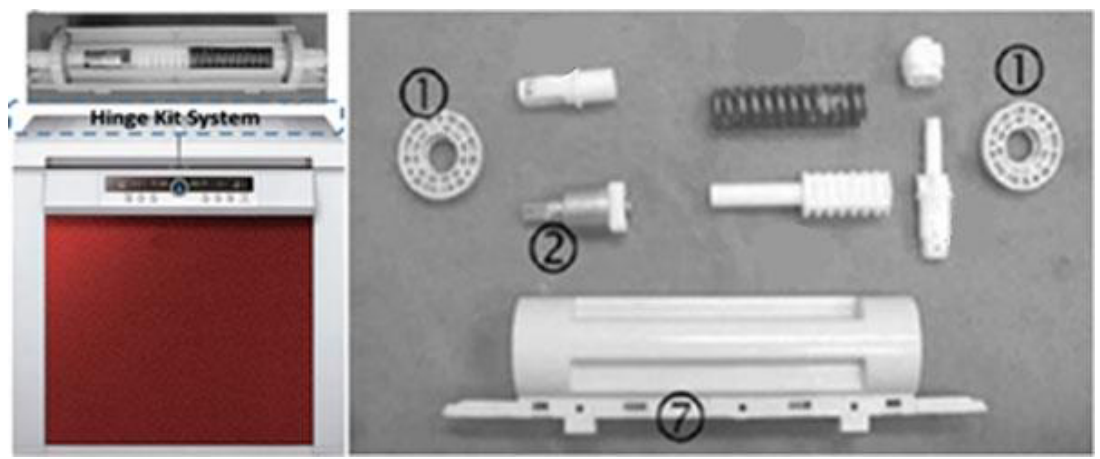

Fig. 4. Domestic refrigerator and HKS parts: kit cover(1), oil damper (2), HKS housing (7). 
When a consumer uses the door in commercially produced refrigerator, they usually want the door to close comfortably. For this (intended) function, the HKS was designed, which should endure the working conditions subjected to it by the consumers who purchase and use the refrigerator. The principal parts in a HKS consists of a kit cover, shaft, spring, and oil damper, etc (See Figure 4). In the marketplace, the HKS parts in refrigerators were failing due to repeated loading under unidentified consumer operation conditions. When data from the marketplace were examined, it appeared that the HKS in the refrigerators had structural design defects, including sharp corner angles and not enough enforced ribs resulting in high stress concentrations. These design flaws, integrated with the repeated impact loads on the HKS, could create a crack in the part and produce a failure in the system.

The closing function of the HKS included several mechanical structural parts. The HKS was often subjected to repeated mechanical impact loads when the consumer closed the door. Door closings required straightforward mechanical procedures: (1) the customers opened the door to take out or store food, and (2) they then closed the door by force. Thus, the HKS were subjected to various loads during the functioning of the refrigerator door. To identify the required $\mathrm{AF}$, it was crucial to determine the forces on the HKS during the operation of the door. Because the HKS was a comparatively simple mechanical structure, the forces impacting the HKS could be modelled with a force-moment equation. As the customer opened or closed the refrigerator door, the stress due to the door weight was concentrated on the HKS.

The stress on the HKS relied on the applied impact due to the elevated weight. The lifestress model (LS model) in Equation (4) can be restated as

$$
T F=A(S)^{-n}=A T^{-\lambda}=A(F \times R)^{-\lambda}
$$

The AF in Equation (5) can be stated as

$$
A F=\left(\frac{S_{1}}{S_{0}}\right)^{n}=\left(\frac{T_{1}}{T_{0}}\right)^{\lambda}=\left(\frac{F_{1} \times R}{F_{0} \times R}\right)^{\lambda}=\left(\frac{F_{1}}{F_{0}}\right)^{\lambda}
$$

The opening and closing of the door ocurred approximately 3 to 10 times per day. With a life design cycle for 10 years, the lifetime of HKS was about 36,500 usage cycles for the worst case. For this scenario, the impact force around the HKS was $1.10 \mathrm{kN}$ which was the anticipated greatest force exerted on the HKS by a user. For the ALT with an accelerated weight, the impact force on the HKS was $2.76 \mathrm{kN}$. Using a cumulative damage exponent, $\lambda$, of 2.0, the AF was established to be roughly 6.3 in Equation (8).

For the lifetime target - B1 life 10 years, the test cycles for sample six pieces using Equation (6) were 23,000 cycles for a shape parameter of 2.0. This parametric ALT was designed to ensure a B1 life 10 years with a $60 \%$ confidence level it would fail less than once during 23,000 cycles. As the door was closing, it could apply to the greatest mechanical impact force necessary to reproduce the accelerated load in the HKS $(2.76 \mathrm{kN})$.

\section{Results and discussion}

Figure 5 show the failed product from the marketplace and the failed from the 1st ALT, respectively. In the 1st ALT, the housing of the HKS fractured at 3,000 cycles and 15,000 cycles. As shown in the picture, the tests affirmed that the HKS housing had a fragile structure near the notch because there were high stresses produced at the sharp edges where it failed. The defective shape of the 1st ALT was very similar to that of the returned product from the marketplace. The corrective action plans for the fragile HKS housing included making fillets, adding enforced ribs, and rounding the notches on the housing of HKS (Figure 6). 

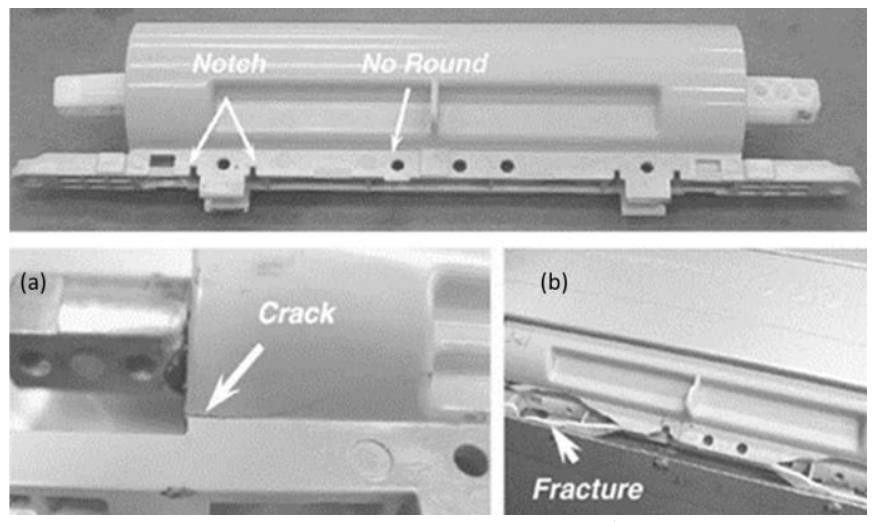

Fig. 5. Failed products from the marketplace and fracture after $1^{\text {st }}$ ALT: (a) marketplace, (b) first ALT

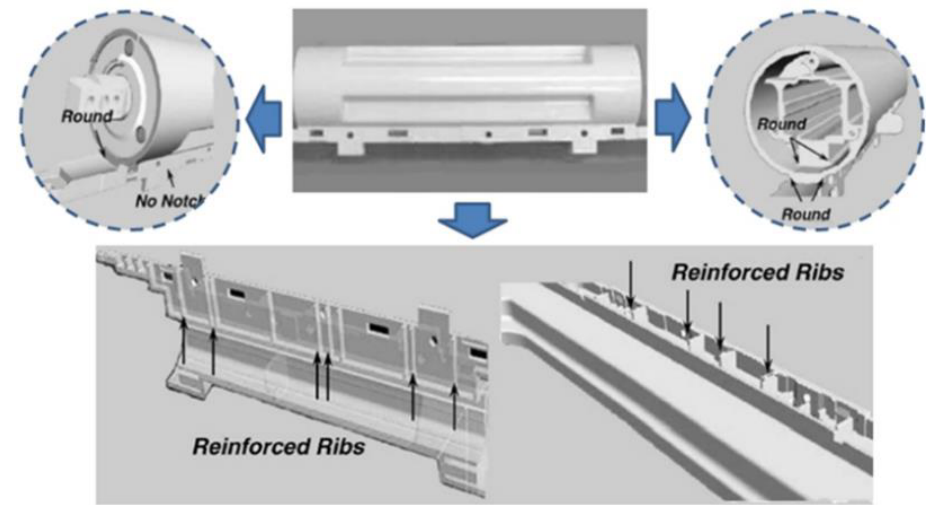

Fig. 6. Redesigned HKS housing structure.

When taking apart the HKS for examination, the leaked oil damper in the HKS was found at 15,000 cycles (Figure 7(a)). When the spilled oil damper was investigated, the sealing structure in the oil damper had a $0.5 \mathrm{~mm}$ gap in the O-ring/Teflon/O-ring assembly. Due to the impact of the door closings, this sealing structure with the gap leaked for first ALT. With the corrective action plan, the sealing structure of the oil damper was modified to have no gap between the Teflon/O-ring/Teflon (Figure 7(b)).

(a)

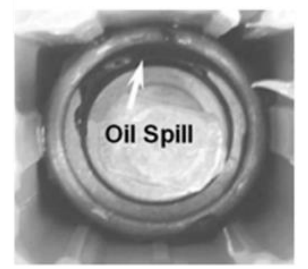

(b)

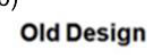

O-ring Assy Gap: $5.2 \mathrm{~mm}$

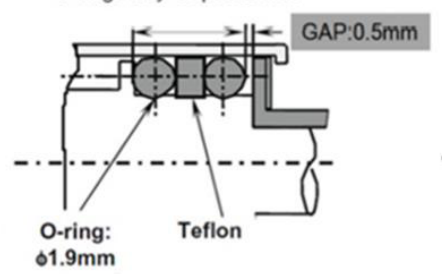

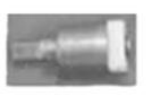

New Design

O-ring Assy Gap: $5.5 \mathrm{~mm}$

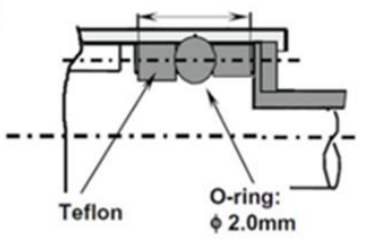

Fig. 7. Redesigned oil damper: (a) Spilled oil damper (b) Redesigned oil damper 

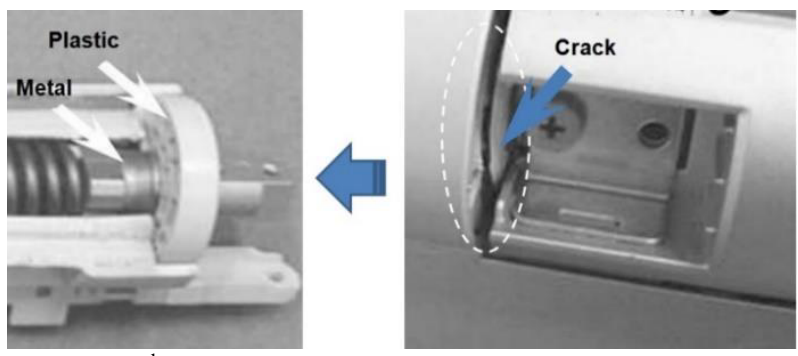

Fig. 8. Failed products after $2^{\text {nd }}$ ALT.

As seen in Figure 8, in the second ALTs, the fracture of the hinge kit cover occurred at $8,000,9,000$, and 14,000 cycles. The root cause of these fractures originated from striking the cover housing (plastic) by the support of oil damper (aluminium). As a corrective action plan, the material of the cover housing were changed from plastic to an Al die-casting. The final design of the HKS could endure the high impact load during operation of the door.

\section{Conclusions}

To enhance the lifetime of a newly designed mechanical system such as HKS, we have proposed a parametric ALT as a systematic reliability method that incorporates: 1) a parametric ALT plan, 2) a load examination, 3) a parametric ALTs with design alterations, and 4) an assessment of the last design needs of the HKS to assure they were fulfilled. A HKS in a refrigerator was investigated as a case study.

- Based on the products that failed both from the marketplace and in 1st ALT, the failure of HKS happened in the fractured HKS housing and oil damper spilling. The design defects of the HKS were the oil sealing structure and the HKS housing that was caused from the concentrated stress due to inappropriate fillets, ribs, and notching. The corrective action plans were the alterations of the HKS housing and the redesigned sealing structure in oil damper.

- Based on the 2nd ALT, the fracturing of HKS happened in the cover housing. The design flaw of the HKS was the material of cover housing. As a corrective action plans, the cover housing from plastic to aluminium was altered. After ALTs, HKS with the correct values for the design parameters were decided to ensure the lifetime target $-\mathrm{B} 1$ life 10 years.

- As systematic reliability design method, we recognized that check of the returned product, load examination, and ALTs with design alternatives was much improved for the newly designed HKS in refrigerator. It also might be relevant to other mechanical systems such as airplane, automobiles, washing machines, and construction equipment. To employ this systematic method, engineers should understand why products fail. In other words, if there are design defects in mechanical product that creates inadequacy of strength (or stiffness) when subjected to repeated loads, the mechanical product will fail over its lifetime.

\section{References}

1. Grove, A. Physics and technology of semiconductor device (Wiley International Edition, 1967)

2. Woo, S., Pecht, M., O’Neal, D. Reliability design and case study of the domestic compressor subjected to repetitive internal stresses, Reliability Engineering \& System Safety, 193, 106604 (2019). 